\title{
Large-Scale Centrifugal Multispinning Production of Polymer Micro- and Nanofibers for Mask Filter Application with a Potential of Cospinning Mixed Multicomponent Fibers
}

\author{
Byeong Eun Kwak, Hyo Jeong Yoo, Eungjun Lee, and Do Hyun Kim* \\ Department of Chemical \& Biomolecular Engineering, Korea Advanced Institute of Science and Technology (KAIST), \\ 291 Daehak-ro, Yuseong-gu, Daejeon 34141, Republic of Korea. \\ Corresponding Author \\ *E-mail: dohyun.kim@kaist.edu.
}

\section{Supporting Information}

\section{Experimental section}

1.1 Materials

Polystyrene (PS; Mw 350,000), poly(methyl methacrylate) (PMMA; Mw 350,000), polyvinylpyrrolidone (PVP; Mw $360,000)$, rhodamine B (>95\%), and chloroform (99.5\%) were purchased from Sigma Aldrich. Ethanol(99.5\%) was purchased from Duksan pure chemicals Co., Ltd. Surgical mask was purchased from HEBio, Corp. Filters with grades of KF80 and KF94 were purchased from Butterfly. Mask consisting of electrospun nanofibers (a grade of KF94) was purchased from FTEnE.

\subsection{Centrifugal multispinning of polymer fiber}

Centrifugal multispinning system was composed of a centrifugal multispinning disk, a motor to rotate the disk, a circular fiber collector consisting of stainless mesh (mesh size of $1 \mathrm{~cm}$ ) with a diameter of $45.5 \mathrm{~cm}$, and threekdScientific LEGATO200 syringe pumps to feed polymer solutions. The multispinning disk has a diameter of $5.5 \mathrm{~cm}$ and is composed of three subdisks which are vertically integrated. Each subdisk has two gaps at the side wall with a size of $1 \mathrm{~mm} \times 80 \mathrm{~mm}$ to eject polymer solution. The bottom subdisk has a shaft at the center which is for connection with motor. The middle and top subdisks have a circular hole at the bottom of each subdisk with diameters of $20 \mathrm{~mm}$ and $30 \mathrm{~mm}$, respectively, and the top subdisk has an additional circular hole at the top of the subdisk with a diameter of $40 \mathrm{~mm}$, for feeding polymer solutions to each subdisk. Typically, different polymer solutions were prepared by dissolving polymer in solvent using sonication method at room temperature. The prepared polymer solutions were fed to each subdisk with a feed flow rate of $5 \mathrm{~mL} / \mathrm{min}$. Rotation speed of the multispinning disk was fixed as 3000 $\mathrm{rpm}$. For all cases, centrifugal multispinning was performed at relative humidity of $35 \%$. To calculate production rate of polymer fibers, spinning was performed for $5 \mathrm{~min}$ twice, and the produced fibers were collected every $5 \mathrm{~min}$, followed by measuring mass of prepared fibers for $10 \mathrm{~min}$.

\subsection{Electrospinning of polymer fiber}

A $1 \mathrm{~mL}$ syringe with a needle of 23 gauge was filled with the prepared polymer solution. The kdScientific LEGATO 200 syringe pump was used for steady flow rate of $0.5,0.7,1,3,5$, and $8 \mathrm{~mL} / \mathrm{h}$. Voltage of $20 \mathrm{kV}$ was applied to syringe tip for fiber production. The electrospun fibers were deposited onto a flat collector and the working distance between syringe tip and collector covered by aluminum foil was $12 \mathrm{~cm}$. The production rate of electrospun fibers was measured by recording the increment of aluminum foil mass every 10 minutes. 
The desired amount of PS nanofibers was equally distributed by hand to whole area $(12 \mathrm{~cm} \times 12 \mathrm{~cm})$ on a nylon mesh (a mesh size of $1 \mathrm{~mm}$ ). Another nylon mesh was covered on the distributed PS nanofibers, followed by compression with a planar weight $(1 \mathrm{~kg})$ for a day. After compression, four edges were masked by tape, and PS nanofiber-based mask filter was fabricated. Examination of water blocking ability was performed under KF-AD test condition. First, $100 \mathrm{~mL}$ of water was filled in $250 \mathrm{~mL}$ beaker, and the test filter was covered the top of the beaker. After that, the beak was reversed for $30 \mathrm{~min}$, and whether the filter can keep $100 \mathrm{~mL}$ of water without spilling or not was examined for $30 \mathrm{~min}$.

\subsection{Characterization}

Scanning electron microscopy (SEM) images were obtained using a FEI company Magellan400, field emission scanning electron microscope. Confocal laser scanning microscopy images were obtained using a Carl Zeiss LSM 880, confocal laser scanning microscope. To visualize nanofibers, blue-emitting carbon dot and rhodamine B were added in PVP and PS solution, respectively, before centrifugal spinning. After cospinning, the prepared multicomponent nanofiber webs were fixed on non-fluorescent carbon tape and covered with cover glass. The fluorescent dyes within multicomponent fiber webs were excited under UV and green light. Contact angle was measured using a SEO Phoenix 300, contact angle analyzer. Electrostatic charge was measured using a Fauser STS1, electrostatic sensor. The measurements were performed with a distance of $5 \mathrm{~mm}$ from the multicomponent fiber webs at relative humidity of $35 \%$. For precision, electrostatic charges were measured at different five points for each fiber web, followed by averaging. Capture efficiency and resistance were obtained using a TSI-8130, automated filter tester. Specifically, sodium chloride $(\mathrm{NaCl})$ aerosol with average particle size of $0.3 \mu \mathrm{m}$ was employed, and flow rate of the aerosolwas fixed at $95 \mathrm{~L} / \mathrm{min}$. Capture efficiency was calculated automatically by comparing the concentration of $\mathrm{NaCl}$ aerosol before and after penetrating test filters as following equation: Capture efficiency $(\%)=\left(\mathrm{C}_{1}-\mathrm{C}_{2}\right) / \mathrm{C}_{1} \mathrm{x} 100$, where $\mathrm{C}_{1}$ and $\mathrm{C}_{2}$ correspond to the aerosol concentrations before and after penetrating filter, respectively. Resistance of filters was also measured at the same aerosol flow rate of $95 \mathrm{~L} / \mathrm{min}$.

\section{Data section}
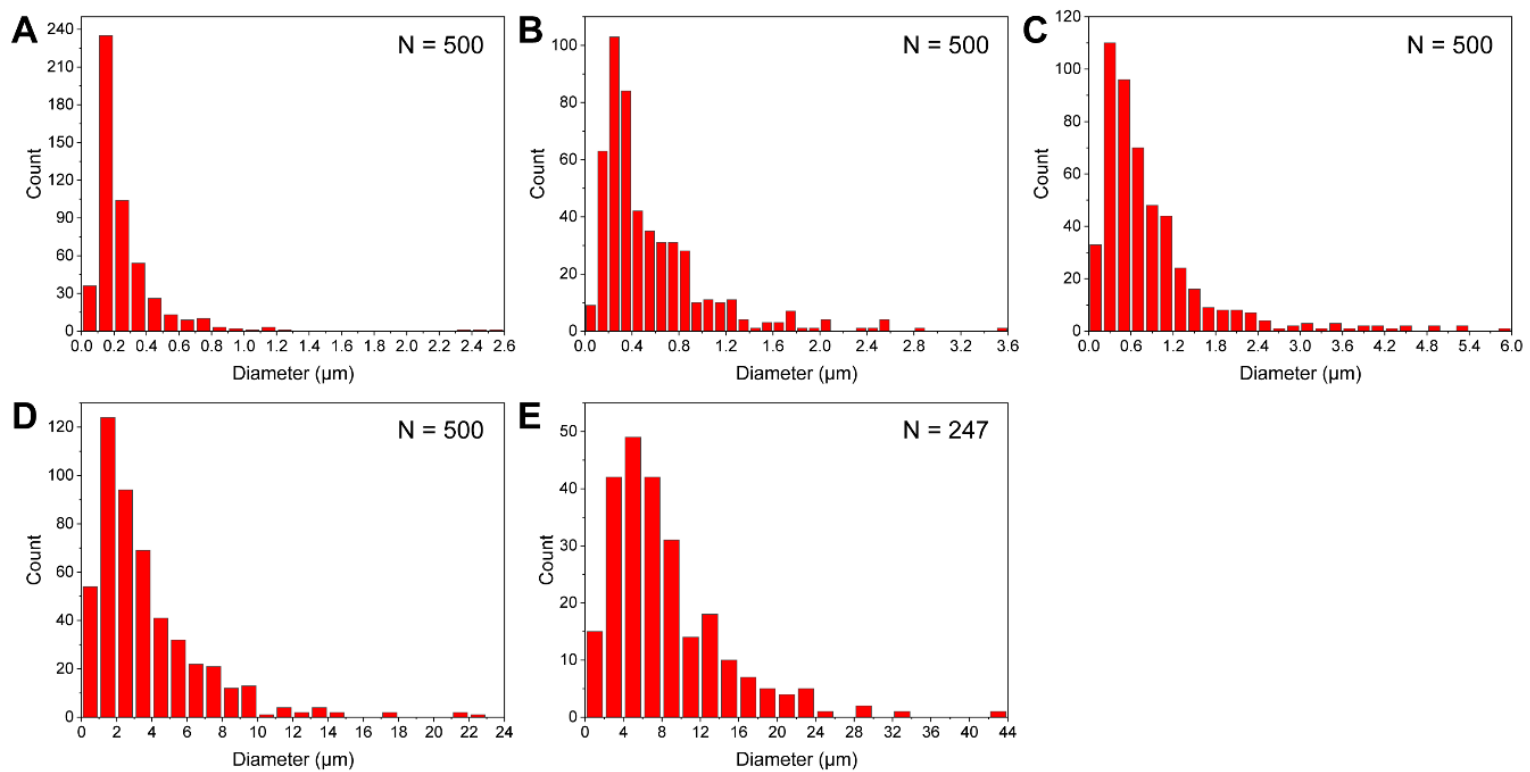

Figure S1. Diameter distributions of PS fibers prepared at polymer concentrations at (A) $3 \mathrm{wt} \%$, (B) $5 \mathrm{wt} \%$, (C) $7 \mathrm{wt} \%$, (D) $10 \mathrm{wt} \%$, and (E) $15 \mathrm{wt} \%$. 

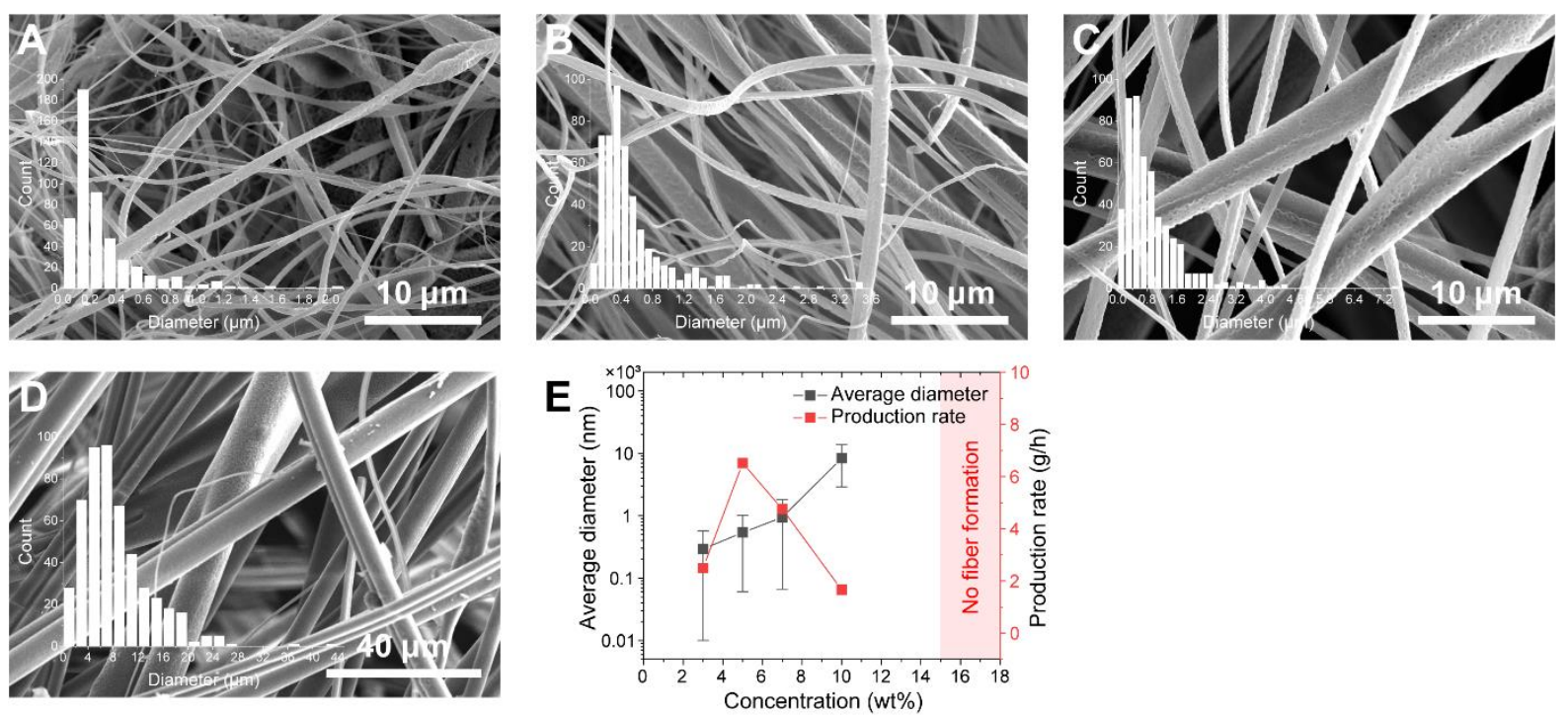

Figure S2. Centrifugal spinning of PMMA fibers. SEM images of prepared PMMA fibers at the concentrations of (A) $3 \mathrm{wt} \%$, (B) $5 \mathrm{wt} \%$, (C) $7 \mathrm{wt} \%$, and (D) $10 \mathrm{wt} \%$. The insets are diameter distributions of the fibers prepared at the corresponding polymer concentrations. (E) Average diameter and production rate of PMMA fibers with different concentrations.
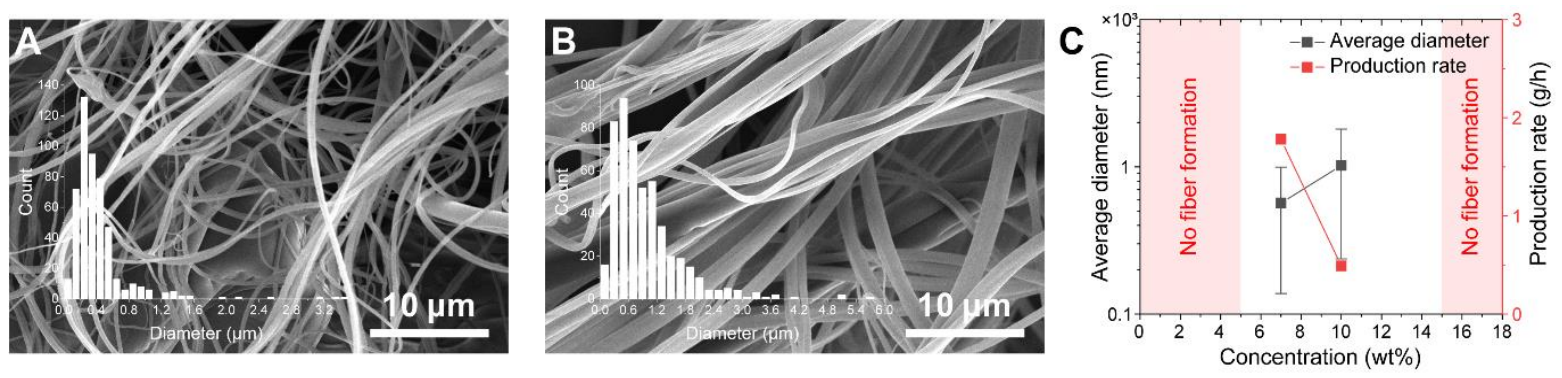

Figure S3. Centrifugal spinning of PVP fibers. SEM images of prepared PVP fibers at the concentrations of (A) $7 \mathrm{wt} \%$ and (B) $10 \mathrm{wt} \%$. The insets are diameter distributions of the fibers prepared at the corresponding polymer concentrations. (C) Average diameter and production rate of PVP fibers with different concentrations.
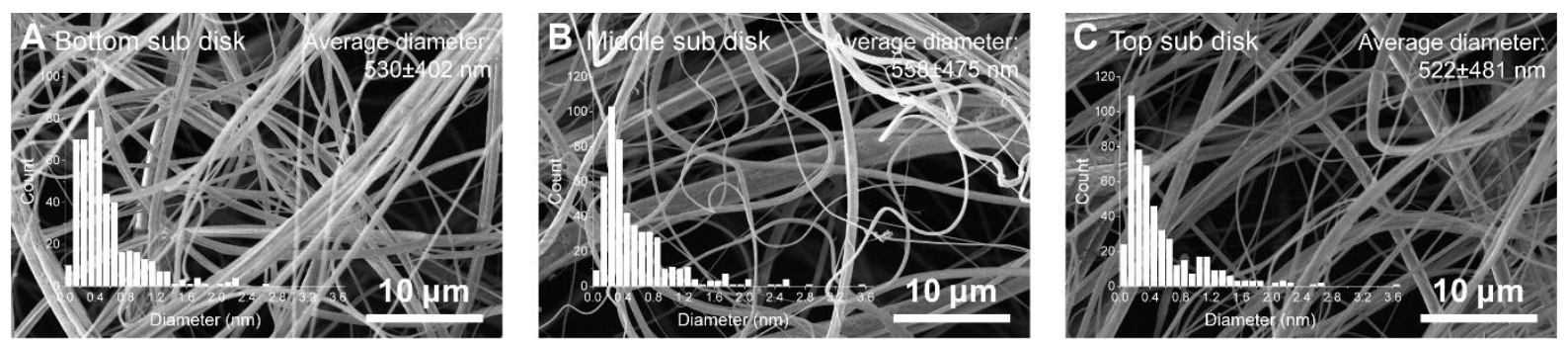

Figure S4. Investigation of the effect of using different subdisks. SEM images of the prepared PS nan ofibers using (A) bottom subdisk, (B) middle subdisk, and (C) top subdisk. The insets are diameter distributions of the fibers prepared using the corresponding subdisk. For spinning, $5 \mathrm{wt} \%$ PS solution was employed, and rotating speed was fixed at $3000 \mathrm{rpm}$. The feed flow rate was set as $5 \mathrm{~mL} / \mathrm{min}$. 

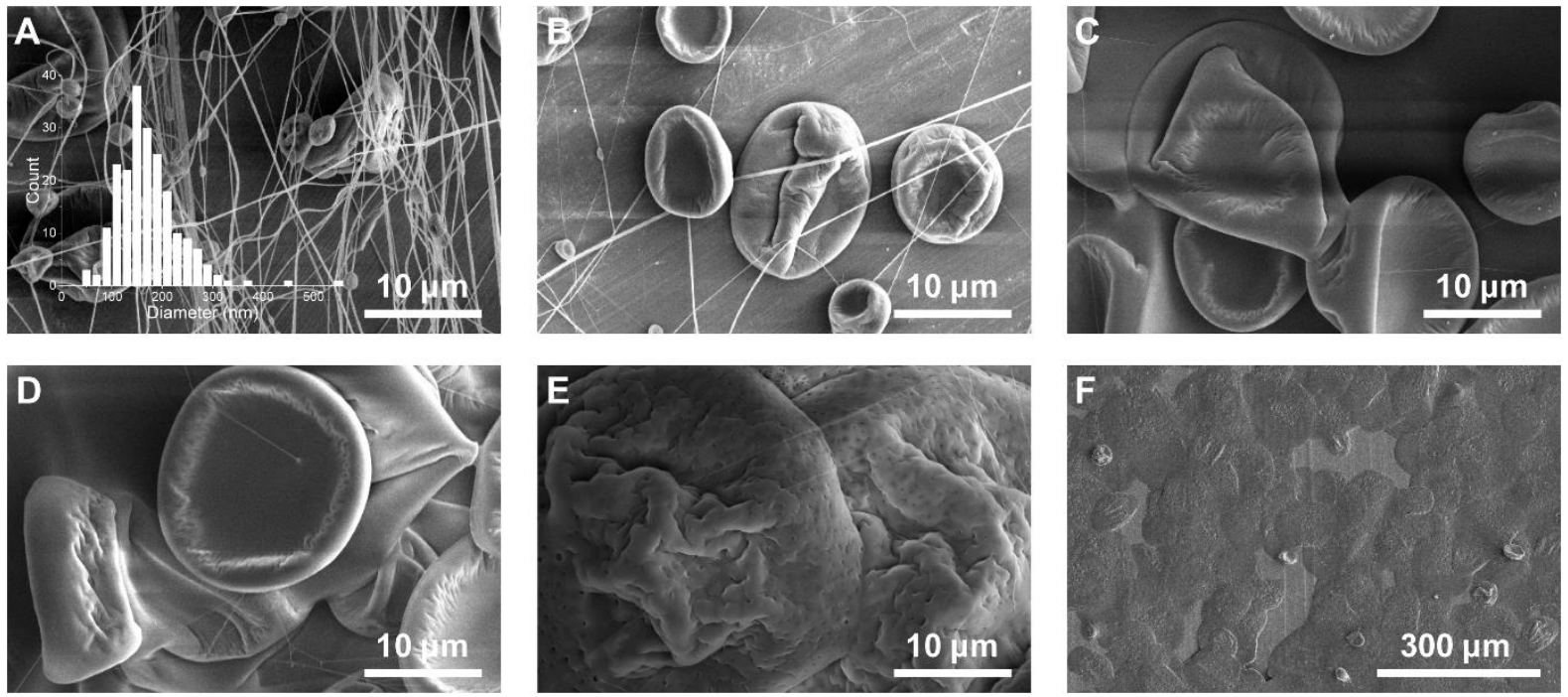

Figure S5. Electrospinning results of PS at the concentration of $3 \mathrm{wt} \%$. SEM images of prepared PS fibers at the concentrations of 3 wt\% with flow rates of (A) $0.5 \mathrm{~mL} / \mathrm{h},(B) 0.7 \mathrm{~mL} / \mathrm{h},(\mathrm{C}) 1 \mathrm{~mL} / \mathrm{h},(\mathrm{D}) 3 \mathrm{~mL} / \mathrm{h},(\mathrm{E}) 5 \mathrm{~mL} / \mathrm{h}$, and (F) $8 \mathrm{~mL} / \mathrm{h}$. The inset is a diameter distribution of the fibers prepared at a flow rate of $0.5 \mathrm{~mL} / \mathrm{h}$.
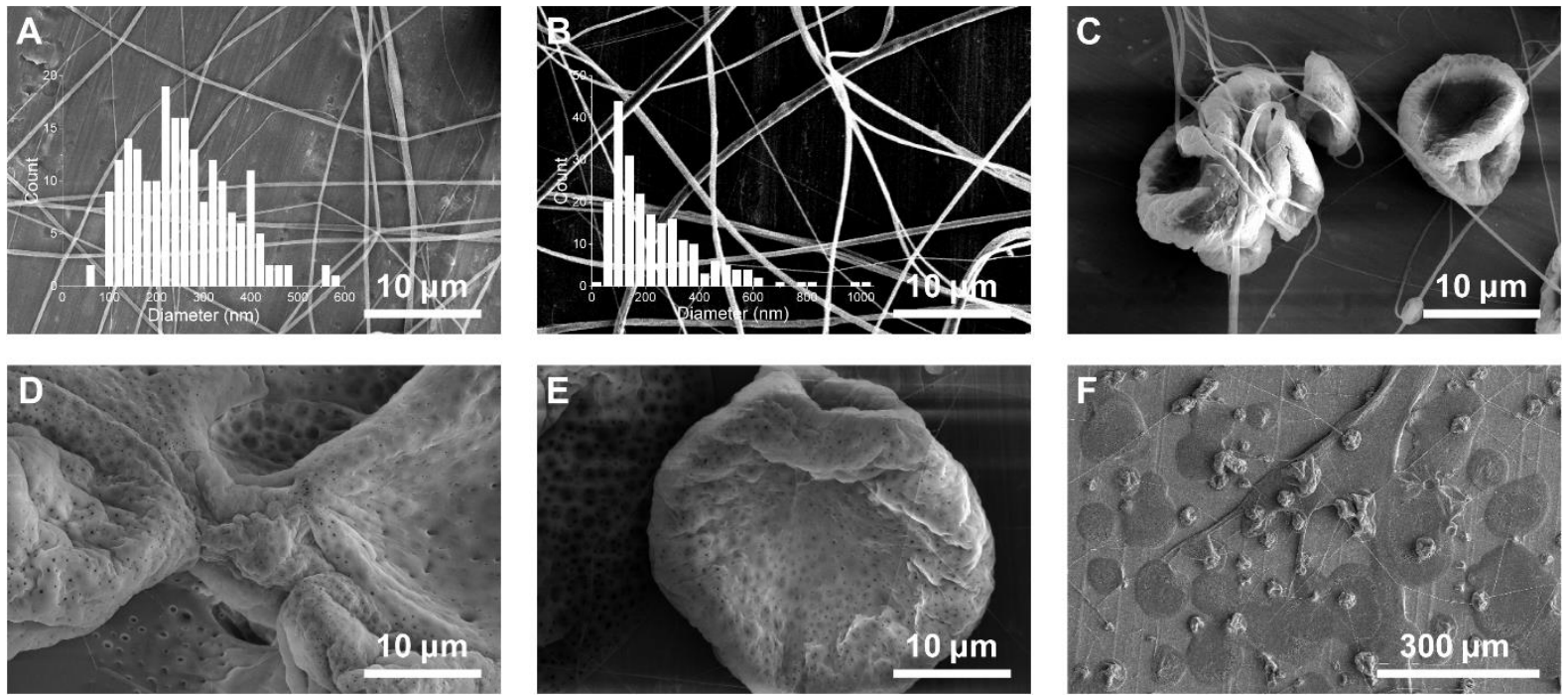

Figure S6. Electrospinning results of PS at the concentration of $5 \mathrm{wt} \%$. SEM images of prepared PS fibers at the concentrations of 5 wt\% with flow rates of (A) $0.5 \mathrm{~mL} / \mathrm{h},(B) 0.7 \mathrm{~mL} / \mathrm{h},(\mathrm{C}) 1 \mathrm{~mL} / \mathrm{h}$, (D) $3 \mathrm{~mL} / \mathrm{h},(\mathrm{E}) 5 \mathrm{~mL} / \mathrm{h}$, and (F) $8 \mathrm{~mL} / \mathrm{h}$. The insets are diameter distributions of the fibers prepared at the corresponding flow rates. 

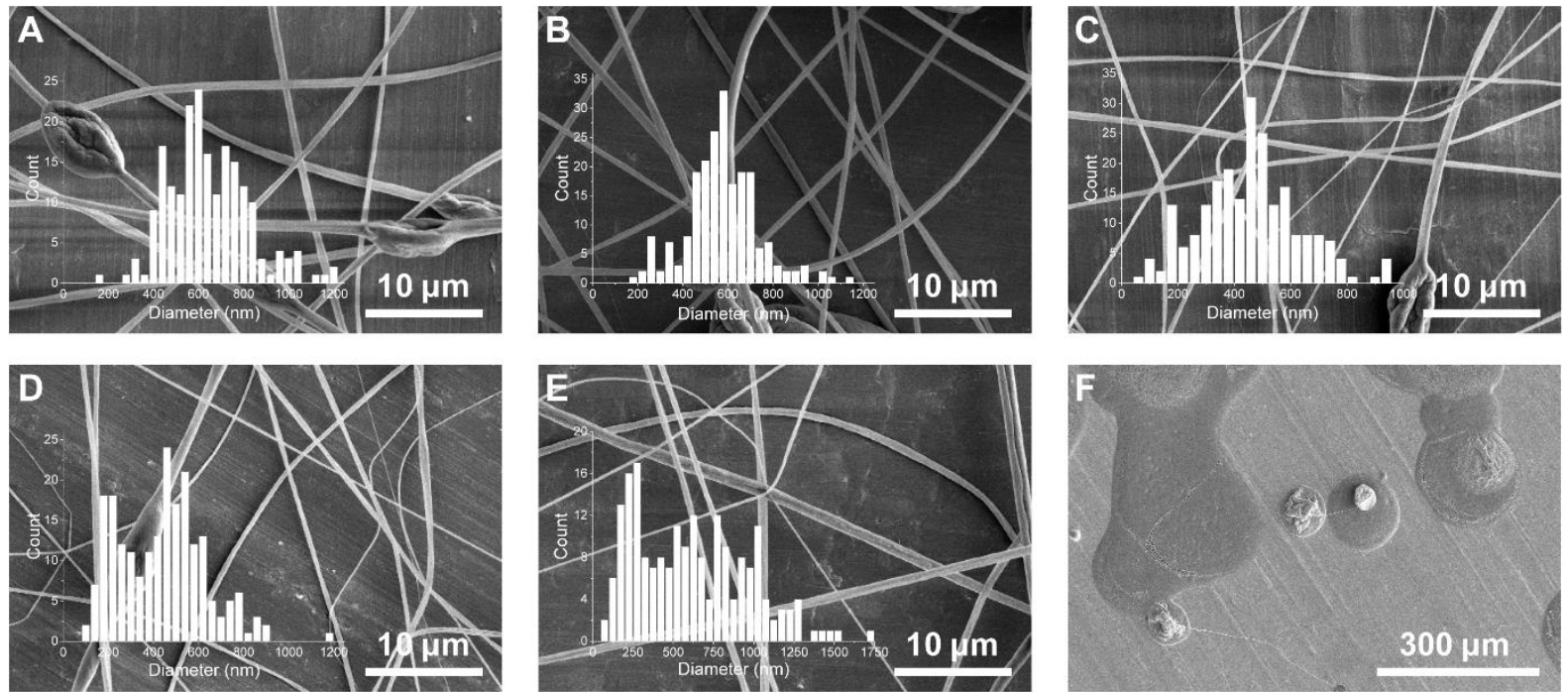

Figure S7. Electrospinning results of PS at the concentration of $7 \mathrm{wt} \%$. SEM images of prepared PS fibers at the concentrations of 7 wt\% with flow rates of (A) $0.5 \mathrm{~mL} / \mathrm{h},(B) 0.7 \mathrm{~mL} / \mathrm{h},(C) 1 \mathrm{~mL} / \mathrm{h}$, (D) $3 \mathrm{~mL} / \mathrm{h},(\mathrm{E}) 5 \mathrm{~mL} / \mathrm{h}$, and (F) $8 \mathrm{~mL} / \mathrm{h}$. The insets are diameter distributions of the fibers prepared at the corresponding flow rates.
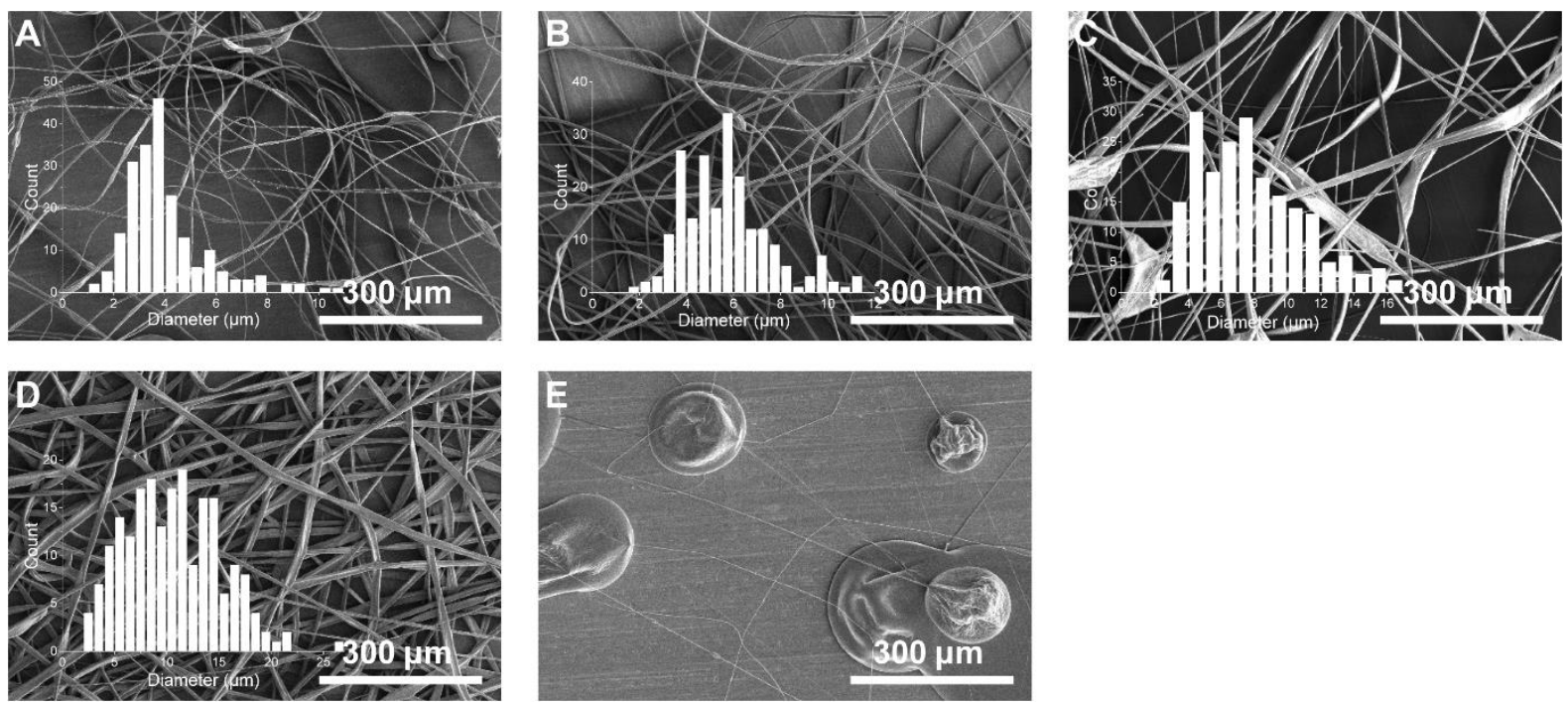

Figure S8. Electrospinning results of PS at the concentration of $10 \mathrm{wt} \%$. SEM images of prepared PS fibers at the concentrations of 10 wt\% with flow rates of (A) $0.7 \mathrm{~mL} / \mathrm{h},(B) 1 \mathrm{~mL} / \mathrm{h},(\mathrm{C}) 3 \mathrm{~mL} / \mathrm{h}$, (D) $5 \mathrm{~mL} / \mathrm{h}$, and (E) $8 \mathrm{~mL} / \mathrm{h}$. The insets are diameter distributions of the fibers prepared at the corresponding flow rates. 

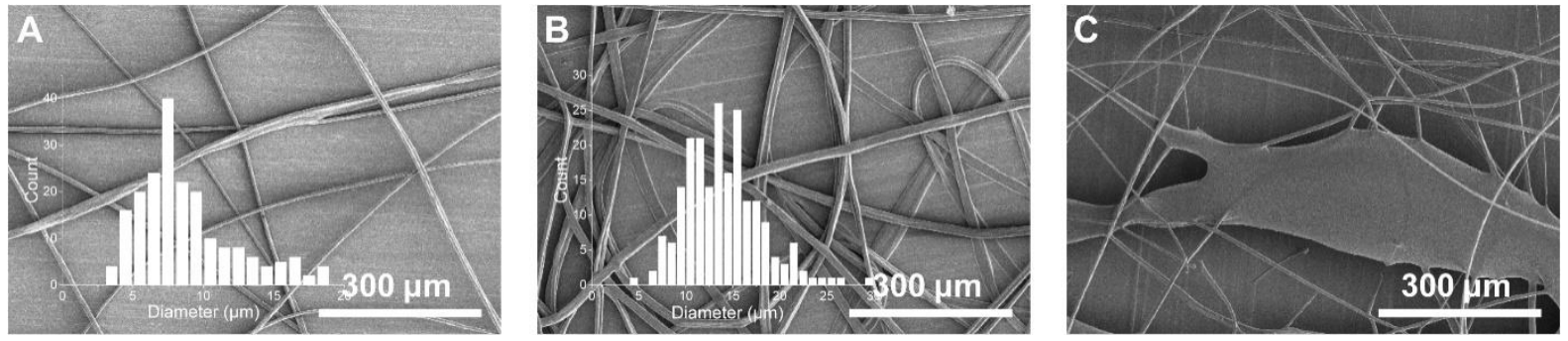

Figure S9. Electrospinning results of PS at the concentration of $15 \mathrm{wt} \%$. SEM images of prepared PS fibers at the concentrations of 15 wt\% with flow rates of (A) $3 \mathrm{~mL} / \mathrm{h},(B) 5 \mathrm{~mL} / \mathrm{h}$, and (C) $8 \mathrm{~mL} / \mathrm{h}$. The insets are diameter distributions of the fibers prepared at the corresponding flow rates.
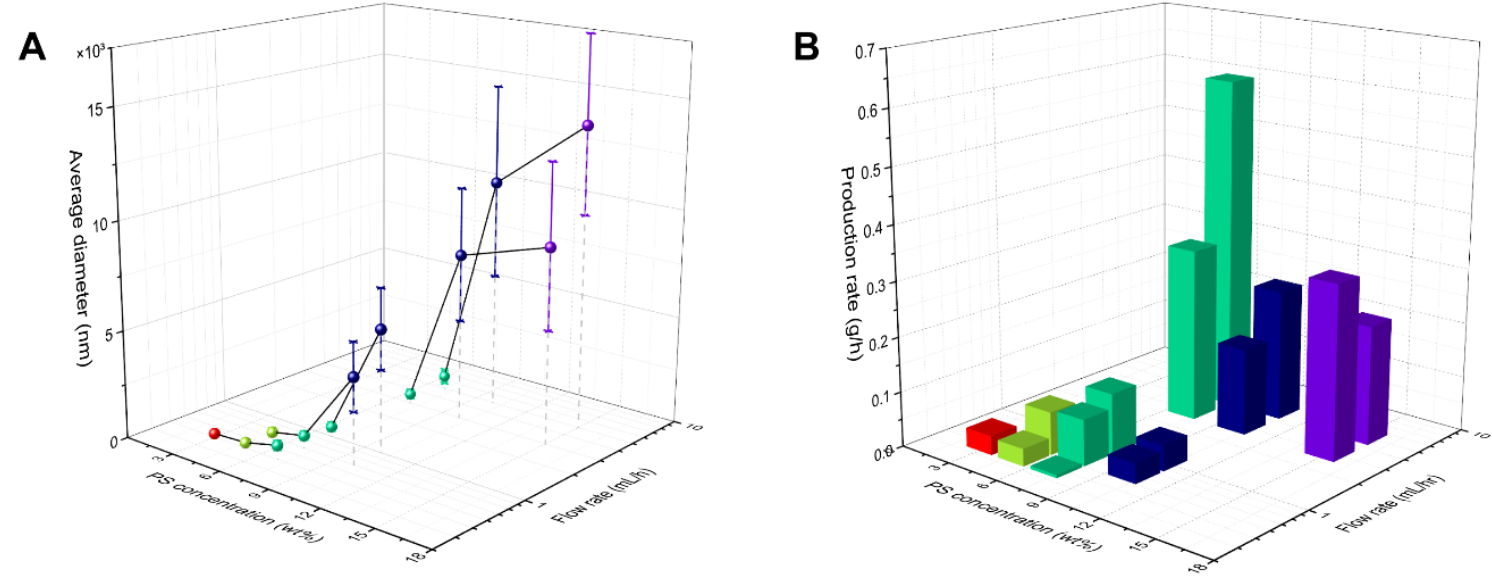

Figure S10. Electrospinning results of PS with different concentrations and flow rates. (A) Average diameter and (B) production rate of PS fibers.
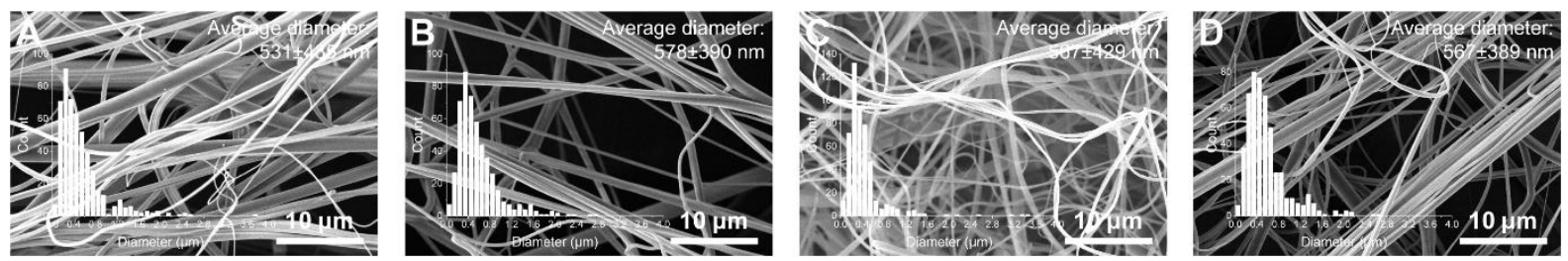

Figure S1 1. Investigation on the effect of flow rate of PVP solution. SEM images of the prepared PVP nanofibers using flow rates of (A) $1 \mathrm{~mL} / \mathrm{min}$, (B) $2.5 \mathrm{~mL} / \mathrm{min}$, (C) $5 \mathrm{~mL} / \mathrm{min}$, and (D) $7 \mathrm{~mL} / \mathrm{min}$. The insets are diameter distributions of the fibers prepared at the corresponding flow rates. 

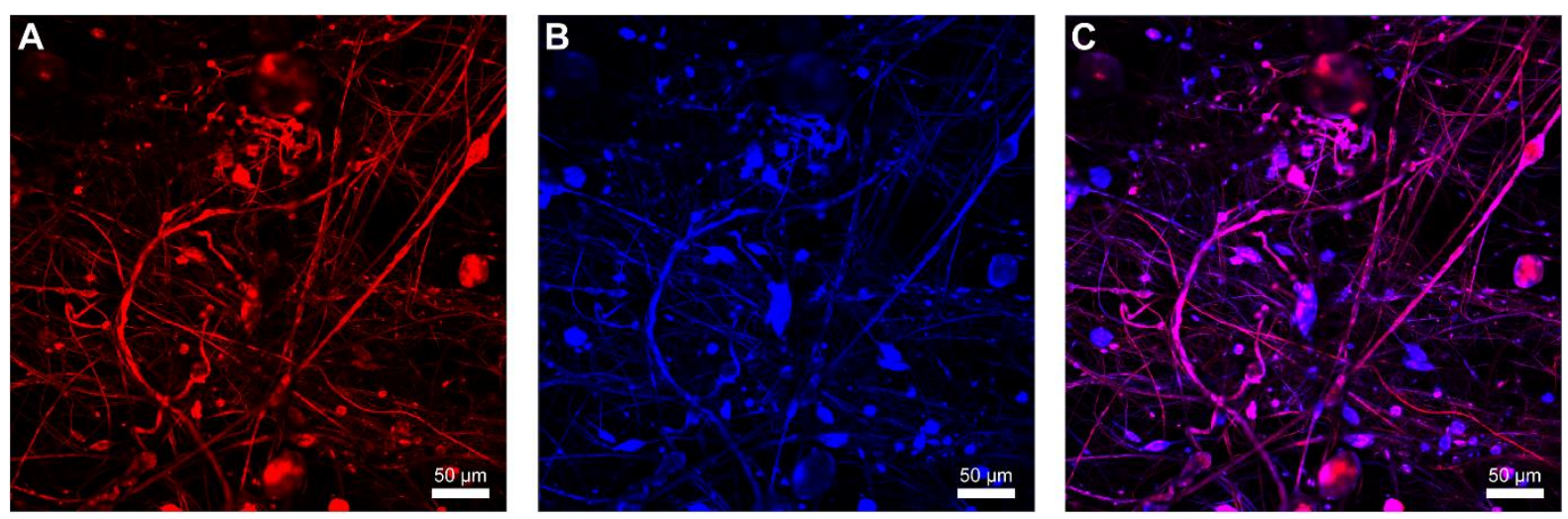

Figure S12. Cospinning results of PS and PVP by centrifugal multispinning at the same subdisk. Confocal laser scanning microscopy images of PS and PVP nan ofibers cospun using the same subdisk under (A) green excitation and (B) UV excitation. (C) Merged image of (A) and (B).

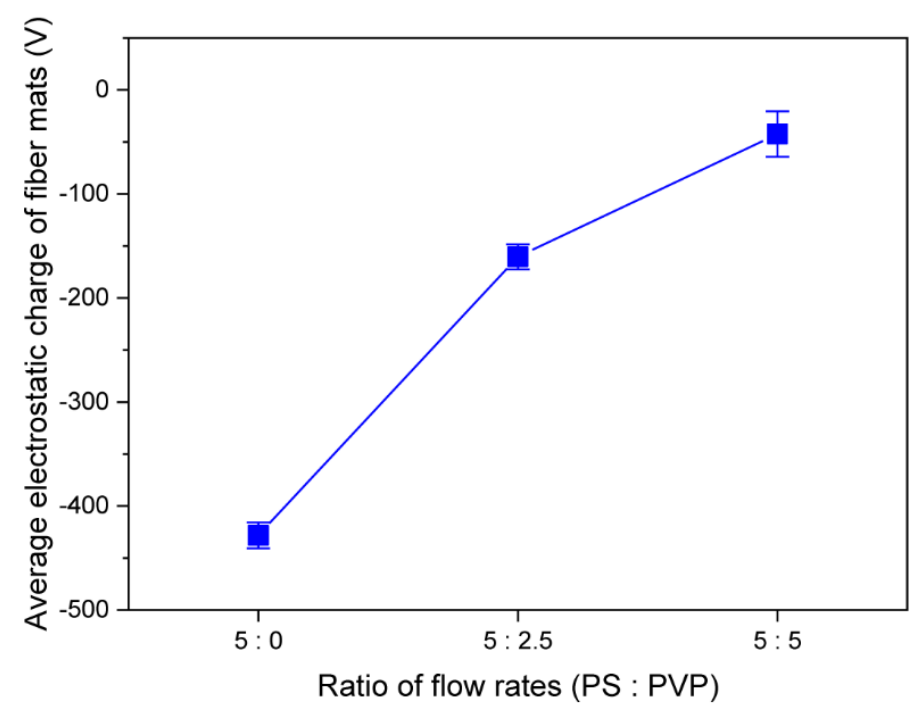

Figure S13. Average electrostatic charge of spun nanofiber webs with different ratios of flow rates of PS and PVP solutions.
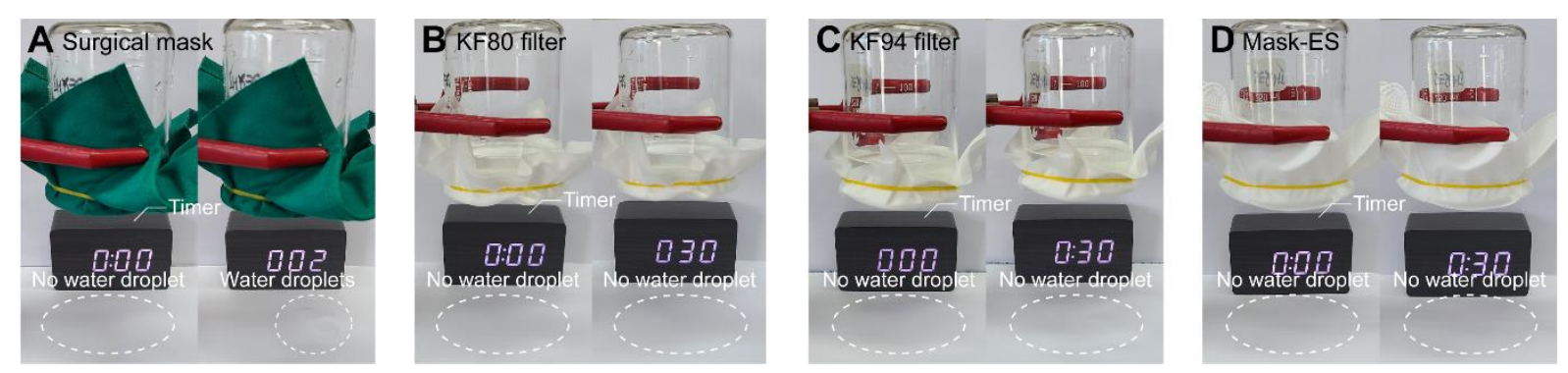

Figure S14. Test for water droplet-blocking ability of commercially available products including (A) surgical mask, (B) KF80 filter, (C) KF94 filter, (D) Mask-ES (KF94 grade) (timer value in min). 
Table S1. Average fiber diameter and production rate of polymer nanofibers spun by mass-producible electrospinning processes and centrifugal multispinning process.

\begin{tabular}{|c|c|c|c|c|c|}
\hline $\begin{array}{l}\text { Refere } \\
\text { nce\# }\end{array}$ & $\begin{array}{l}\text { Spinning } \\
\text { method }\end{array}$ & Spinneret & Polymer species & $\begin{array}{l}\text { Average fiber } \\
\text { diameter (nm) }\end{array}$ & $\begin{array}{c}\text { Production rate } \\
(\mathrm{g} / \mathrm{h})\end{array}$ \\
\hline 29 & $\begin{array}{c}\text { Needleless } \\
\text { electrospinning } \\
\text { (ES) }\end{array}$ & Annular spinneret & $\begin{array}{l}\text { Polyacrylonitrile } \\
\text { (PAN) }\end{array}$ & $\begin{array}{c}\sim 200^{1} \\
(133.07 \pm 21.66- \\
351.69 \pm 40.98)\end{array}$ & 4.5 \\
\hline 30 & Multijet ES & Porous tube & Nylon 6 & $170.6 \pm 75.0$ & 5 \\
\hline 31 & Multinozzle ES & Four-hole spinneret & $\begin{array}{l}\text { Poly(ethylene oxide) } \\
\text { (PEO) }\end{array}$ & $\sim 220-240^{2,3}$ & $1.68(\sim 0.42 /$ hole $)$ \\
\hline 32 & Multinozzle ES & $\begin{array}{c}\text { Porous hollow tube ( } 20 \\
\text { holes) }\end{array}$ & $\begin{array}{l}\text { Polyvinylpyrrolidone } \\
\text { (PVP) }\end{array}$ & $\sim 300-600^{2,3}$ & $\begin{array}{c}0.3-0.5(\sim 0.02- \\
0.03 / \text { hole })\end{array}$ \\
\hline 33 & Multinozzle ES & $\begin{array}{l}\text { Concentric pipes ( } 10 \\
\text { pipes) }\end{array}$ & $\begin{array}{l}\text { Poly(vinyl alcohol) } \\
\text { (PVA) }\end{array}$ & $\mathrm{N} / \mathrm{A}$ & $\sim 0.06 /$ pipe \\
\hline 34 & Needleless ES & Cylinder & PAN & $\sim 350^{1}$ & $\sim 8.6^{1}$ \\
\hline 35 & Needleless ES & Cylinder & PVA & $\sim 350^{1}$ & $\sim 8.2^{1}$ \\
\hline 36 & Needleless ES & Bead wire (200 beads) & PVP & $200-650^{2,3}$ & $40(\sim 0.20 /$ bead $)$ \\
\hline 37 & Syringeless ES & $\begin{array}{l}\text { Rotating probed cylinder } \\
\text { (12 probes) }\end{array}$ & $\begin{array}{l}\text { Thermoplastic } \\
\text { polyurethane }\end{array}$ & $\mathrm{N} / \mathrm{A}$ & $3.2(\sim 0.27 /$ probe $)$ \\
\hline 38 & Needleless ES & Rings (4 rings) & PVA & $\sim 325^{1,3}$ & $\sim 9.5^{1}$ ( 2.4/ring) \\
\hline 39 & Needleless ES & Linear flume spinneret & PVA & $108-210^{2,3}$ & 4.85 \\
\hline 40 & Needleless ES & Spiral coil (5 spirals) & PAN & $\sim 250^{1}$ & 23 ( 4.6/spiral) \\
\hline 41 & Needleless ES & Twisted wire & PVP & $397 \pm 76$ & 5.23 \\
\hline 42 & Needleless ES & $\begin{array}{l}\text { Moving conventional } \\
\text { yarn }\end{array}$ & PAN & $409 \pm 60$ & 1.17 \\
\hline 43 & Multinozzle ES & 19 nozzles & PEO & $\sim 250-475^{2,3}$ & $\begin{array}{c}0.618-0.712 \\
(\sim 0.0325- \\
0.0375 / \text { nozzle })\end{array}$ \\
\hline 44 & Needleless ES & Bead structure nozzle & PAN & $420^{3}$ & 0.918 \\
\hline 45 & Multinozzle ES & $\begin{array}{l}\text { Tubes with holes ( } 16 \\
\text { holes per } 2 \text { tubes) }\end{array}$ & SU-8 & $200-500^{2,3}$ & $0.75(0.047 /$ hole $)$ \\
\hline 46 & Bubble ES & Double-nozzle spinneret & PAN & $\begin{array}{c}236 \text { (standard } \\
\text { deviation: } 14 \% \text { ) }\end{array}$ & $\sim 7.2^{1}(\sim 3.6 /$ nozzle $)$ \\
\hline 47 & Syringeless ES & Needle-disk (24 needles) & $\begin{array}{l}\text { Poly(vinylidene } \\
\text { fluoride-co-hexa- } \\
\text { fluoropropylene) }\end{array}$ & $245.23 \pm 33.75$ & $\begin{array}{c}13.5 \\
(\sim 0.563 / \text { needle })\end{array}$ \\
\hline 48 & $\begin{array}{c}\text { Splashing } \\
\text { needleless ES }\end{array}$ & Metal roller & $\mathrm{PEO}$ & $\sim 100-400^{2,3}$ & $12-14$ \\
\hline 49 & Multinozzle ES & $\begin{array}{l}\text { Plastic filter with holes ( } 3 \text {, } \\
\text { 5, } 7 \text { holes) }\end{array}$ & $\mathrm{PEO}$ & $225^{3}$ & $\sim 2^{1}(\sim 0.3 /$ hole $)$ \\
\hline 50 & Needleless ES & Conical wire coil & PVA & $193 \pm 85-424 \pm 243^{2}$ & $\sim 2.75$ \\
\hline $\begin{array}{l}\text { This } \\
\text { work }\end{array}$ & $\begin{array}{l}\text { Centrifugal } \\
\text { multispinning }\end{array}$ & $\begin{array}{l}\text { Multispinning disk with } \\
\text { triple subdisks }\end{array}$ & Polystyrene & $558 \pm 475$ & $\sim 25(\sim 8.3 /$ subdisk $)$ \\
\hline
\end{tabular}

All the values are based on the condition at maximum production rate; 1: only can be found in graph; 2 : only diameter range was provided (average diameter was not provided at maximum production rate condition); 3: standard deviation was not provided; 
Table S2. Average fiber diameter and production rate of polymer nanofibers spun by mass-producible electrospinning processes and centrifugal multispinning process.

\begin{tabular}{|c|c|c|c|c|c|c|c|c|}
\hline $\begin{array}{c}\text { Refere } \\
\text { nce\# }\end{array}$ & $\begin{array}{l}\text { Spinning } \\
\text { method }\end{array}$ & $\begin{array}{l}\text { Polymer } \\
\text { species }\end{array}$ & $\begin{array}{l}\text { Average } \\
\text { fiber } \\
\text { diameter } \\
(\mathbf{n m})\end{array}$ & $\begin{array}{l}\text { Capture } \\
\text { efficiency } \\
(\%)\end{array}$ & $\begin{array}{l}\text { Resistance } \\
\quad(\mathbf{P a})\end{array}$ & $\begin{array}{c}\text { Test } \\
\text { aerosol }\end{array}$ & $\begin{array}{c}\text { Face } \\
\text { velocity }\end{array}$ & Note \\
\hline \multirow[b]{2}{*}{61} & \multirow[b]{2}{*}{$\begin{array}{c}\text { Electrospin } \\
\text { ning (ES) }\end{array}$} & $\begin{array}{l}\text { Polyacrilonit } \\
\text { rile (PAN) }\end{array}$ & $\begin{array}{l}293.7- \\
612.8^{2}\end{array}$ & $57.73-81.98$ & $106-125$ & \multirow{2}{*}{$\begin{array}{c}\text { Potassiu } \\
\text { m } \\
\text { chloride } \\
(\mathrm{KCl} \text {; } \\
0.3 \mu \mathrm{m})\end{array}$} & \multirow[b]{2}{*}{$15.92 \mathrm{~cm} / \mathrm{s}$} & $\begin{array}{l}\text { Areal mass: } \\
4.2 \mathrm{~g} / \mathrm{m}^{2}\end{array}$ \\
\hline & & Polyamide 6 & $128.0^{2}$ & 99.41 & 241 & & & $\begin{array}{c}\text { Nanofiber } \\
\text { net; areal } \\
\text { mass: } 2.1 \\
\mathrm{~g} / \mathrm{m}^{2}\end{array}$ \\
\hline 62 & ES & PAN & $\begin{array}{c}630-1350^{2} \\
\text { (scaffold } \\
\text { fiber) } / 20 \\
\text { (nanonet) }\end{array}$ & 99.998 & 110 & $\begin{array}{c}\text { Particula } \\
\text { te matter } \\
\text { with a } \\
\text { diameter } \\
\text { of } 0.3 \\
\mu \mathrm{m} \\
(\mathrm{PM} 0.3)\end{array}$ & $5.33 \mathrm{~cm} / \mathrm{s}$ & Nanonet \\
\hline \multirow[b]{2}{*}{63} & \multirow[b]{2}{*}{ ES } & PAN & $\begin{array}{l}175 \pm 11- \\
558 \pm 14\end{array}$ & 40.806-98.89 & $3-53$ & \multirow{2}{*}{$\begin{array}{c}\text { Sodium } \\
\text { chloride } \\
(\mathrm{NaCl} \text {; } \\
0.3-0.5 \\
\mu \mathrm{m})\end{array}$} & $\mathrm{N} / \mathrm{A}$ & - \\
\hline & & $\begin{array}{l}\text { PAN/fluorin } \\
\text { ated } \\
\text { polyurethane } \\
\text { (PU) }\end{array}$ & $\begin{array}{c}558 \pm 14 / 6 \\
98 \pm 23\end{array}$ & $\sim 99.9^{1}$ & $\begin{array}{c}\sim 115^{1} \\
\left(\sim 320^{1} \text { at } 90\right. \\
\mathrm{L} / \mathrm{min})\end{array}$ & & $32 \mathrm{~L} / \mathrm{min}$ & $\begin{array}{l}\text { Basis weight: } \\
24.04 \mathrm{~g} / \mathrm{m}^{2}\end{array}$ \\
\hline 64 & ES & $\begin{array}{c}\text { Poly(vinyl } \\
\text { chloride)/P } \\
U\end{array}$ & $960^{2}$ & 99.5 & $\begin{array}{c}144\left(\sim 400^{1}\right. \\
\text { at } 14.1 \mathrm{~cm} / \mathrm{s})\end{array}$ & $\begin{array}{c}\mathrm{NaCl} \\
(0.3-0.5 \\
\mu \mathrm{m})\end{array}$ & $\begin{array}{c}5.3 \text { and } \\
14.1 \mathrm{~cm} / \mathrm{s}\end{array}$ & $\begin{array}{l}\text { Basis weight: } \\
20.72 \mathrm{~g} / \mathrm{m}^{2}\end{array}$ \\
\hline \multirow{2}{*}{65} & \multirow{2}{*}{ ES } & \multirow{2}{*}{ PAN } & $\begin{array}{l}67 \pm 25- \\
858 \pm 63\end{array}$ & $73.8-96.6$ & $172.3-566.1$ & \multirow{2}{*}{$\mathrm{PM}_{0.3}$} & \multirow{2}{*}{$5 \mathrm{~cm} / \mathrm{s}$} & - \\
\hline & & & $549 \pm 67$ & 78.2 & 566.1 & & & $\begin{array}{l}\text { Basis weight: } \\
25.38 \mathrm{~g} / \mathrm{m}^{2}\end{array}$ \\
\hline \multirow{3}{*}{66} & \multirow{3}{*}{$\begin{array}{l}\text { Solution } \\
\text { blow } \\
\text { spinning }\end{array}$} & $\begin{array}{l}\text { Cellulose } \\
\text { diacetate }\end{array}$ & $950 \pm 410$ & $62-76$ & $53-63$ & \multirow{3}{*}{$\mathrm{PM}_{2.5}$} & \multirow{3}{*}{ N/A } & $\begin{array}{l}\text { Weight } \\
\text { basis: } \\
1.7 \times 10^{-3}- \\
2.7 \times 10^{-3} \\
\mathrm{~g} / \mathrm{cm}^{2}\end{array}$ \\
\hline & & PAN & $590 \pm 180$ & $62-92$ & $40-50$ & & & $\begin{array}{l}\text { Weight } \\
\text { basis: } \\
1.0 \times 10^{-4} \text { - } \\
3.2 \times 10^{-4} \\
\mathrm{~g} / \mathrm{cm}^{2}\end{array}$ \\
\hline & & $\begin{array}{l}\text { Poly(vinylide } \\
\text { ne fluoride) }\end{array}$ & $660 \pm 240$ & $63-74$ & $60-70$ & & & $\begin{array}{l}\text { Weight } \\
\text { basis: } \\
1.7 \times 10^{-3}- \\
6.1 \times 10^{-3} \\
\mathrm{~g} / \mathrm{cm}^{2}\end{array}$ \\
\hline $\begin{array}{l}\text { This } \\
\text { work }\end{array}$ & $\begin{array}{l}\text { Centrifugal } \\
\text { multispinn } \\
\text { ing }\end{array}$ & Polystyrene & $558 \pm 475$ & 82.97 & 374 & $\begin{array}{c}\mathrm{NaCl} \\
\text { (average } \\
\text { diameter }\end{array}$ & $\begin{array}{c}15.83 \mathrm{~cm} / \mathrm{s} \\
(95 \\
\mathrm{L} / \mathrm{min})\end{array}$ & $\begin{array}{l}\text { Nanofiber } \\
\text { weight: } 0.02 \\
\mathrm{~g} / \mathrm{cm}^{2}\end{array}$ \\
\hline
\end{tabular}




\begin{tabular}{|l|l|l|c|c|c|c|c|}
\hline & & & 89.59 & 504 & $\begin{array}{c}\text { of } 0.3 \\
\mu \mathrm{m})\end{array}$ & $\begin{array}{c}\text { Nanofiber } \\
\text { weight: } 0.03 \\
\mathrm{~g} / \mathrm{cm}^{2}\end{array}$ \\
\cline { 4 - 7 } & & & 96.63 & 743 & $\begin{array}{c}\text { Nanofiber } \\
\text { weight: } 0.04 \\
\mathrm{~g} / \mathrm{cm}^{2}\end{array}$ \\
\hline
\end{tabular}

All the values are based on the condition at maximum production rate; 1: only can be found in graph; 2: standard deviation was not provided; 